\title{
A conceptual and graphical method for converging multisubject behavioral observational data into a single process indicator
}

\author{
WILLIAM A. GRIFFIN \\ Arizona State University, Tempe, Arizona
}

\begin{abstract}
Typically, in observational research, each behavior of a subject is assigned a code that represents theoretically relevant and contextually sensitive events. These discrete behavioral acts are then strung together to denote a process. With multiple interactants, the investigator has two choices: ignore subject interdependence and analyze as if the data strings are discrete, or treat the strings as interdependent and create a summary index that represents the combined codes across subjects. This article illustrates a method of combining discrete within-subjects behavioral indicators into behavior-specific domains and then conglomerating the disparate domain strings across subjects in such a way that the aggregate reflects the observed process. This aggregation is represented by a coordination index, expressed as either interactant disparity or coherence. Also, animation and visualization methods are presented that illustrate how social processes can be modeled by using either the coordination index or the separate domain values.
\end{abstract}

Investigators use observational coding to record the behavior of humans and animals. Typically, each behavior is coded, and then the codes are strung together to show the behavior sequence exhibited by the subject. This code sequence provides two vital pieces of information about behavior: its occurrence and its timing. From this record, sequential patterns are discerned and analyzed. Searching for sequential information with this strategy has a long and productive history (Cairns, 1979; Lamb, Suomi, \& Stephenson, 1979; Sackett, 1978). Yet, since its inception, investigators using this method have acknowledged that the most interesting questions about behavior focus on interactional processes, not on the individual behavior of an organism (Bakeman \& Gottman, 1997).

Historically, tracking the sequential behavior of an individual organism was done simply because it was easier than trying to capture the complex, joint behavior of two or

This article is based on a presentation given at the Second International Conference on Methods and Techniques in Behavioral Research: Measuring Behavior ' 98 , sponsored by Noldus Information Technology, August 1998. This work was supported by National Institute of Mental Health Grant 1 RO1 MH51 184-01A1. I thank Amy Decker-Haas, Roger Bakeman, Carol Martin, and Vicenç Quera for their numerous and insightful comments on this work. Special thanks are due Helene Barcelo for her help in simplifying the mathematics and Simon Windmill at Windmill Fraser Multimedia, Inc., for his help in simplifying the animation scripts. Send Samba inquiries to J. T. Stasko at the Georgia Institute of Technology, College of Computing (e-mail: stacko@)cc.gatech. edu or $<$ http://www.cc.gatech.edu/gvu/softviz/algoanim/ samba.html $>$ ). A copy of the Samba animation file and the animations for 2- and 3subject interactions (.avi format), along with their graphed indices, are available from the author. Correspondence concerning this article should be sent to W. A. Griffin, Marital Interaction Laboratory, Department of Family Resources and Human Development, YHE 2502, Arizona State University, Tempe, AZ 85287 (e-mail: william.griffin@a asu.edu). more organisms. To describe a joint process, investigators would typically code each individual organism and then attempt to reconstruct the process through some data analytic strategy (e.g., sequential analysis) or structural procedure (e.g., matrix analysis). This has worked well for simple processes (see Martin \& Bateson, 1996, for numerous techniques). Unfortunately, such a basic strategy fails to capture the essence of social processes, because interaction among organisms is intricately organized and coordinated. Coding individuals and then reassembling their behavior strings to create a facsimile of interaction seems inadequate for the increasingly complex theories and models of behavior (Newell \& Molenaar, 1998; see, also, Fogel, Lyra, \& Valsiner, 1997, or van Geert, 1994). Fogel (1993), for example, posits that behaviors are coregulated, not exchanged. He persuasively argues that individual behavior occurs because of the interaction, not vice versa; that is, individual behavior occurs because of what is occurring between the interactants (Fogel, 1993). Behavior is social and dynamic for Fogel and other contemporary theorists (see, e.g., Bernieri, Reznick, \& Rosenthal, 1988); consequently, the quantification of process is increasingly important.

The current predicament for behavioral observationalists is how to form a sufficiently information-rich process index from traditionally constructed individual data streams. Ideally, this composite index should contain the individual subject's contribution to the state and yet reflect the larger process. This is a bottom-up perspective (i.e., individual $\rightarrow$ process). Viewed from a top-down perspective, the objective is to capture the process and know exactly how each individual contributed to that process. Irrespective of viewpoint, two features of the interaction need assessing: the process and the individual. Several notable 
attempts to capture this complexity is evident in the work of Tronick, Brazleton, and colleagues (see, e.g., Als, Tronick, \& Brazelton, 1979). Their work entails overlaying time-based microcoded behaviors with qualitative impressions of the interactants. These strings are resynthesized and interpreted as a dynamic whole. Unfortunately, it is very complex, and although the microcoding is quantified, the composite impressions that form the basis for interpretation are not completely quantifiable. Another attempt in this area has come from Hinde (Hinde \& Herrmann, 1975; Hinde \& Stevenson-Hinde, 1987), who, for several decades, has attempted to construct methods for quantifying and demonstrating the dynamics of behavioral interaction. After coding the individual behaviors of the interactants, Hinde uses numerous methods to portray the dynamics of the assessed system; these range from ratios of the interactants' behaviors to behavior proportions across interactions (see Hinde, 1997, for an overview). Although inventive and informative, the methods are complex, difficult to interpret, and, essentially, a compilation rather than an integration of the behaviors of each individual in the interaction.

This article introduces a method of aggregating intrasubject data strings into behavior states that, when pooled across subjects, can quantify complex interorganism processes. Each interactant contributes to an index that expresses the process. This index is appropriate for traditional statistical analysis. Fortunately, being a complex multicomponent index, it has the capacity to reconstruct a visual representation of the observed process. Consequently, I also illustrate how the index can be incorporated into a research strategy that uses visualization and animation techniques, at least at the exploratory stage, to understand better the quantified processes.

\section{CODING BEHAVIOR}

\section{Information Saliency}

Any attempt to reconstruct complex social processes must initially address the issue of information saliency. Essentially, the investigator must determine what occurs during an interaction that is necessary to reproduce accurately the process. Ideally, investigators want to take the greatest amount of data available and reduce it to a much smaller, more manageable set of salient behavioral indicators with minimum loss of information. These indicators, in turn, should lend themselves to reconstitution as parameters of a process.

The first inclination is to code everything that occurs and then find a method of combining the data. Even if it were possible to integrate the data to get a composite portrayal of the process, it would probably be too cumbersome and error producing, and, most likely, it would be unnecessary. Other disciplines, most notably ethology and computer science, have found that a few simple bits of relevant information are sufficient to reproduce complex processes. For example, ethologists have shown that very simple algorithms specifying behavioral reactions to the near neighbor can explain very complex behaviors in schools of fish or flocks of birds (see, e.g., Shaw, 1970). Similarly, computer scientists, using a few very simple parameters to characterize interactions among objects, are able to produce complex animations that appear to mimic natural processes (Reynolds, 1987), such as bicyclists racing over various types of terrain (Brogan \& Hodgins, 1997). These seemingly distant disciplines actually complement each other. For the one, a few well-selected data points predict complex natural phenomena, and for the other, a few well-constructed algorithms produce outputs that mimic natural processes. By assuming that similar data parsimony rules apply for other social phenomena, then, relative to the objective of this article, coded salient data reduced according to an efficient algorithm should be able to reproduce the observed behavioral process. Saliency is determined by the data's ability to reproduce the phenomena being studied. To acquire the relevant codes, the behavioral scientist either develops an ethogram of the phenomena or else selects theoretically pertinent codes (i.e., behaviors). Either method produces a set of behaviors used to model the observed processes. The objective is to create models that accurately reproduce the process with a minimum number of codes. Codes remaining in a parsimonious yet actuate model are considered salient. The investigator decides, a priori, the features of the interaction or process that are theoretically relevant, assessable by the observer, and reliably codable. For example, in mother-infant interactions, the mother's facing the child is important to synchronization. Clearly, mother attentiveness - coded as discrete elements (head orientation, eye gaze, body position)-are necessary indicators that the investigator must accurately code to assess the mother's behavior during this process (Fogel, 1993).

Modeling social processes after having observed and coded multiple subjects requires at least four steps according to the proposed method. First, code the behavior of individual interactants; these codes are the behavioral indicators. Second, the behavioral indicators then collapse into specific behavioral domains; these domains represent unique aspects or dimensions of the subject. Next, the behavioral domain data reduce into a single index; collectively, they represent the behavioral state of the individual. Finally, the behavioral process emerges by combining the behavioral states among all the interactants. Figure 1 schematically illustrates these steps. The initial step in this process is coding the individual.

\section{Behavioral Indicators}

Every social process has defining features. These features also foreshadow the process trajectory. The objective is to capture the sequential description of these features shown by each individual. I refer to these salient features as behavioral indicators.

A code is assigned to each indicator, and each subject is described by $n$ codes at time $t_{x}$. These codes reflect an observable or inferred disposition of the subject during 


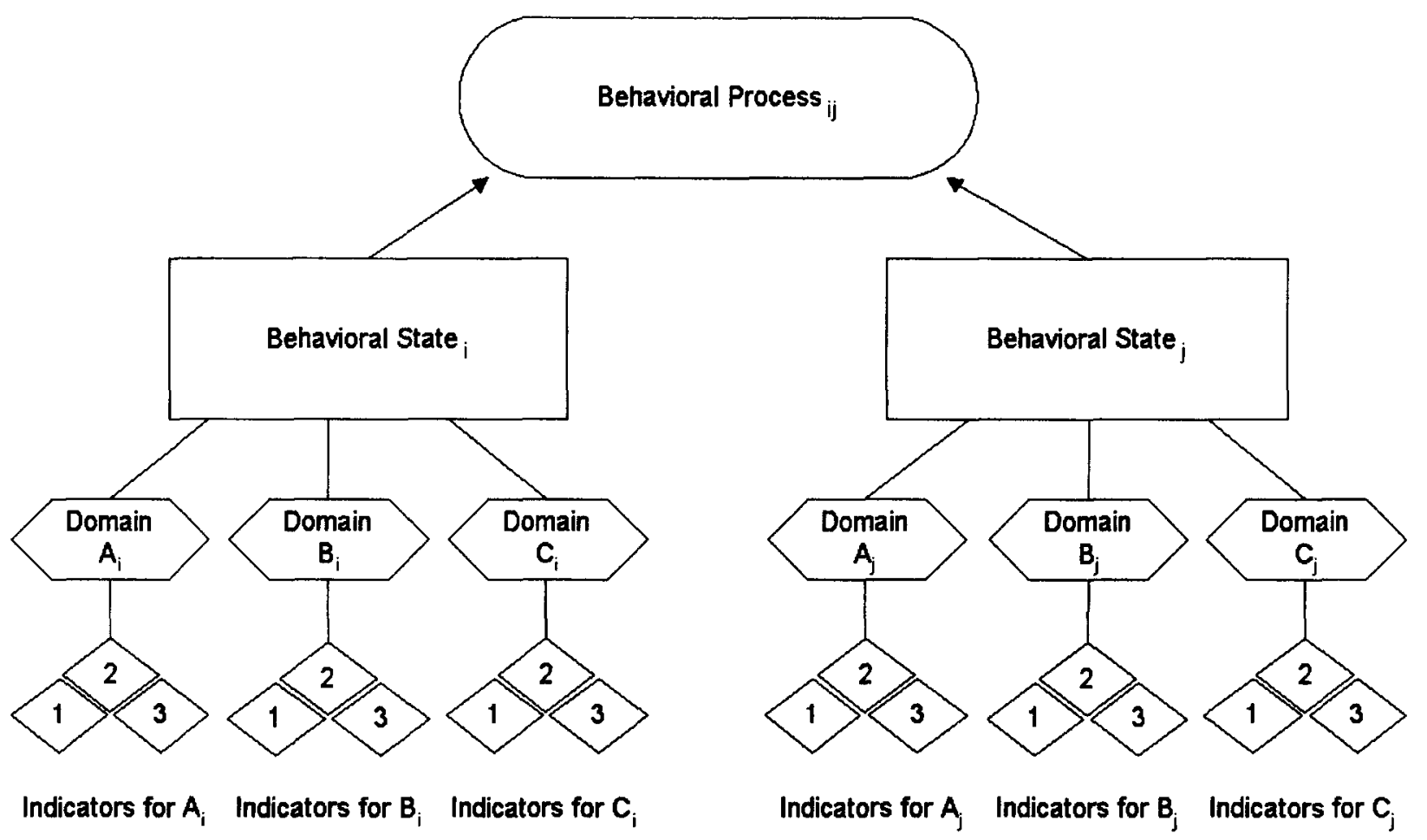

Figure 1. The progression from behavioral indicators (i.e., typical intrasubject codes) to behavioral domains to behavioral states. States join to form intrasubject processes.

the unitized time period. It is assumed that each code (indicator) is scored with minimal nonsystematic error (i.e., coded by an objective coder against a criterion); hence, any differences between subjects for any specific indicator at $t_{x}$ reflects true differences. If each code is reliable (i.e., adequate kappa [Cohen, 1960] from long data strings), then, in general, the obtained difference value is reliable. Sequential observational data that are not reliable introduce special and unwanted complications for the data analysts (see Rogosa \& Ghandour, 1991), especially in the use of sequential analysis (see Gardner, 1995).

Determining indicators. At the behavioral indicator level, the investigator makes several important decisions. First, the investigator needs to decide which major behavior domains (i.e., dimensions) effectively capture the action of the subject that is pertinent to the theory or model. These can be determined empirically or theoretically. In human studies, these might include verbal behavior, nonverbal behavior, body position, location, and, possibly, physiological data. It is critical to remember that these data points must be relevant to the process of interest, not simply data reflecting the individual. For example, if one were studying dyadic interaction in humans, behaviors associated with speaker and listener roles would be important, because the process occurs as a composite of these respective behaviors. Moreover, assume that codes are divided into verbal and nonverbal modes across listener and speaker roles. In the speaker role, for example, the following codes are applicable: verbal agreement, negative statement, talk, and problem solution; for the listener, back channeling, head nod, eye gaze, eye roll, and interrupt are applicable codes. Numerous existing coding systems-at least, for dyads (mother-child, husbandwife) and families-contain similar indicator codes (see Markman \& Notarius, 1987).

After selecting the relevant domains, the researcher must determine how many different indicators of the domain are codable. The objective is to be inclusive-the more codes the better. For example, again assume that the investigator is interested in human dyadic interaction and that the listener's monitoring behavior is an important dimension of interaction. Assume further that the investigator can reliably code three indicators of this domain: head nod, back channel, and eye gaze. From these three codes, the investigator captures the nonverbal or paralinguistic features of the monitoring dimension of the process. Note that this example covers only one behavioral domain; a good coding scheme would have many domains, each ideally having multiple indicators.

Scoring the indicators. Next, the investigator needs to determine whether to score each indicator as nominal or as quantitative. This decision is important, because it determines how the indicators eventually collapse to form the behavioral domain index. Table 1 illustrates the simple construction of scores for a single subject across nominal and quantitative indicators with positive and 
Table 1

Simulated Data of a Single Subject Showing Indicator Values Across Five Nominal and Two Quantitative Codes Over Eight Time Units

\begin{tabular}{|c|c|c|c|c|c|c|c|}
\hline \multirow[b]{4}{*}{ Time Unit } & \multicolumn{7}{|c|}{ Behavior Indicators } \\
\hline & \multicolumn{5}{|c|}{ Nominal } & \multicolumn{2}{|c|}{ Quantitative } \\
\hline & \multicolumn{3}{|c|}{$\begin{array}{l}\text { Positive } \\
\text { Domain }\end{array}$} & \multicolumn{2}{|c|}{$\begin{array}{l}\text { Negative } \\
\text { Domain }\end{array}$} & \multirow{2}{*}{$\begin{array}{c}\text { Positive } \\
\text { Domain } \\
\text { (A) }\end{array}$} & \multirow{2}{*}{$\begin{array}{c}\text { Negative } \\
\text { Domain } \\
\text { (B) }\end{array}$} \\
\hline & $\mathrm{A}$ & $B$ & $\mathrm{C}$ & A & $\mathrm{B}$ & & \\
\hline 1 & 1 & 0 & 0 & l & 0 & 5 & 3 \\
\hline 2 & 1 & 1 & 1 & 0 & 0 & 2 & 3 \\
\hline 3 & 0 & 1 & 0 & 1 & 0 & 4 & 1 \\
\hline 4 & 1 & 1 & 0 & 1 & 1 & 1 & 2 \\
\hline 5 & 1 & 1 & 1 & 1 & 0 & 3 & 3 \\
\hline 6 & 0 & 0 & 1 & 1 & 1 & 2 & 2 \\
\hline 7 & 0 & 0 & 0 & 1 & 1 & 2 & 1 \\
\hline 8 & 1 & 0 & 1 & 1 & 0 & 4 & 4 \\
\hline
\end{tabular}

negative valences. This is typical of the data format used in most observational laboratories to represent subject behavior over $n$ time units.

Each method of scoring a behavior has advantages and disadvantages. Nominal scoring, although restrictive to a dichotomous outcome (e.g., on/off; present/not present), allows the investigator to meaningfully tally the score values over a domain. For example, if each indicator within a domain is scored in the same direction (i.e., good behavior $=1$, bad $=0$ ), a simple sum provides an informative index. If the number of indicators exceeds three or four and each is in the same direction, proportion also provides a good summary index. Similarly, if the domain has indicators that reflect contradictory behaviors or valences (e.g., listener head nod vs. listener eye roll), an interpretable summary index is constructed using difference, absolute difference, odds (i.e., ratio), ${ }^{\prime}$ or (In)odds, where $\ln$ is the natural logarithm (i.e., $\log$ to the base $e ; 2.71828$ ). ${ }^{2}$ Interpretation of the differences or odds, of course, depends on the construction of the underlying codes.

An illustration of various combinations and their results is given in Table 2. Note that each combinatorial method produces a range of values. The best index to use depends on indicator format, construction, and clarity of interpretation relative to the research question. If, for example, the investigator thinks that the effects of the presence or the absence of a behavior is additive, the simple sum score across indicators is sufficient. On the other hand, if the same assumption is phrased in the form of the proportion of codes that have a specific valence, proportion is a good index of the behavior. An example of this can be found in the recent work of Gottman (1994). He simply subtracts the positive codes from the negative codes at each floor switch (i.e., talk turn), plots this value, and uses the slope of the line to classify couples along a dimension of dyadic affect regulation.

Similarly, depending on the research question, the use of odds or $\ln$ (odds) adds additional interpretive value to the index. For example, the odds can be interpreted as the likelihood of a code relative to another code; in effect, for example, it conveys information about the odds of a positive valence code(s) (e.g., head nod, back channel) relative to negative valence (e.g., eye roll) at $t_{x}$ (see note 2 ).

A quantitative indicator, reflecting a rating or scale, provides additional information not available with nominal scoring. However, quantitatively scored indicators are difficult to combine meaningfully with other indicators, either nominal or quantitative. Thus, quantitative indicators usually represent a single domain (e.g., negative affect level). Although it is possible to have two quantitative indicators of the same behavioral domain, they should be contradictory or, at least, different enough that their differences or odds are interpretable. For example, dividing a positive and a negative rating during the same time unit estimates the odds index of these valences.

Indicator weighting. Finally, it is possible to weight any indicator or indicator summary (either nominal or quantitative) score, justifying the weight by theory or past experience with the data. For example, assume that the domain of interactant listener consists of head nod, back channel, eye gaze, and eye roll. All the indicators except eye roll are considered good facilitators of conversation; hence, the best summary index would be a difference score or an odds (i.e., ratio) between the three good indicators

Table 2

An Illustration of Possible Domain Values From Various Combinatorial Methods, Using Indicator Data From Table 1

\begin{tabular}{|c|c|c|c|c|c|c|c|c|c|c|}
\hline \multirow[b]{3}{*}{ Time Unit } & \multicolumn{8}{|c|}{ Methods With Nominal Indicators } & & \\
\hline & \multicolumn{2}{|c|}{$\begin{array}{l}\text { Positive } \\
\text { Valence }\end{array}$} & \multicolumn{2}{|c|}{$\begin{array}{l}\text { Negative } \\
\text { Valence }\end{array}$} & \multirow[b]{2}{*}{ Diff } & \multirow[b]{2}{*}{ Abs Diff } & \multirow[b]{2}{*}{ Odds* } & \multirow[b]{2}{*}{ Ln (Odds) $\dagger$} & \multicolumn{2}{|c|}{$\begin{array}{c}\text { Methods With } \\
\text { Quantitative Indicators }\end{array}$} \\
\hline & Sum & Pro & Sum & Pro & & & & & Odds & Ln(Odds) \\
\hline 1 & 1.00 & 0.33 & 1.00 & 0.50 & 0.00 & 0.00 & 1.00 & 0.00 & 1.67 & 0.51 \\
\hline 2 & 3.00 & 1.00 & 0.00 & 0.00 & 3.00 & 3.00 & 7.00 & 1.95 & 0.67 & -0.41 \\
\hline 3 & 1.00 & 0.33 & 1.00 & 0.50 & 0.00 & 0.00 & 1.00 & 0.00 & 4.00 & 1.39 \\
\hline 4 & 2.00 & 0.67 & 2.00 & 1.00 & 0.00 & 0.00 & 1.00 & 0.00 & 0.50 & -0.69 \\
\hline 5 & 3.00 & 1.00 & 1.00 & 0.50 & 2.00 & 2.00 & 2.33 & 0.85 & 1.00 & 0.00 \\
\hline 6 & 1.00 & 0.33 & 2.00 & 1.00 & -1.00 & 1.00 & 0.60 & -0.51 & 1.00 & 0.00 \\
\hline 7 & 0.00 & 0.00 & 2.00 & 1.00 & -2.00 & 2.00 & 0.20 & -1.61 & 2.00 & 0.69 \\
\hline 8 & 2.00 & 0.67 & 1.00 & 0.50 & 1.00 & 1.00 & 1.67 & 0.51 & 1.00 & 0.00 \\
\hline
\end{tabular}

Note-Pro, proportion; Diff, difference; Abs Diff, absolute difference. $* .5$ constant added (see text). ${ }^{+} \operatorname{Ln}=$ natural $\log$. 
and eye roll. Assume that all four indicators were scored 1 at $t_{x}$, producing a difference score of $2(3-1)$ or an odds of $3(3 / 1)$. The utility of this score assumes, of course, that positive and negative listener behavior has equivalent impact on a conversation. Available evidence indicates that the detrimental effect that negative behavior has on a conversation is greater than the effect that positive behavior has in improving a conversation (Noller, 1984). This is especially true for eye roll, which has a contemptuous connotation in marital dyadic interaction (Gottman, 1994). Thus, the investigator can justify weighting the negative indicator. In this example, given that three positives are possible (one for each good behavior), a weighting of 3 or 4 for eye roll could be justified. In the above example, with an eye roll occurrence equal to 4 , the difference score becomes $-1(3-4)$ or the odds are $.75(3 / 4)$, both indicating that negative predominates for listener behavior at $t_{x}$. It is also possible to weight same-valence behaviors differentially within the same domain. For example, if back channel has been shown to be more influential (i.e., changes the conversation trajectory) than other similar valence codes within the same domain, it can be weighted in proportion to its expected influence.

\section{Behavioral Domains}

A behavioral domain is a multicode composite index representing a unique dimension or feature of the interactant. Each domain depicts a feature of the interactant observed for its empirical, theoretical, or general putative value. When the investigator determines which domains are important, he or she simultaneously decides which behaviors make up the domain.
Although it is possible to have a single, usually quantitative, indicator for a domain, most often the investigator wants numerous indicators per domain, because each domain is an inclusive description of a specific aspect or dimension of the interactant. Table 3 illustrates a simple example of four domains (nonverbal positive: head nod, back channel, eye gaze; nonverbal negative: eye roll; verbal negative; verbal positive: agree, problem solution) with 3 subjects $(i, j, k)$ over four time units $\left(t_{0}-t_{3}\right)$. The time unit here is arbitrary; it could reflect talk turns or discrete intervals (e.g., 5 or $1 \mathrm{sec}$ ). All the indicators are nominal $(0 / 1)$, except verbal negative, which is a rating with a range of $1-5$. Table 3 shows how the individual indicator scores produce the domain index.

As can be seen in Table 3, behavioral domains represent a substantial reduction in data; in this simple data set, the four domain indices represent seven coded behaviors, a $43 \%$ reduction in data. If verbal negative were composed of several nominal indicators instead of the quantitative score, the reduction would be greater. Although every behavioral domain index is subsequently collapsed into the behavioral state score, each independently represents a specific behavioral feature and, as such, can be used in traditional sequential or time series analyses (see, e.g., Griffin \& Gottman, 1990).

It is assumed that, at the indicator level, reliability has been obtained, with either Cohen's kappa (Cohen, 1960) or some other commonly used index of reliability (see Suen \& Ary, 1989, for an overview). If each indicator has acceptable reliability, the domain formed from the individual indicators is reliable. This assumption does not imply that the domain is reliable in the test construction

Table 3

An Illustration of Domain and State Index Construction at Four Time Periods for 3 Subjects Across Multiple Indicators, Domains, and Valences

\begin{tabular}{|c|c|c|c|c|c|c|c|c|c|c|c|}
\hline \multirow[b]{4}{*}{ Time } & \multirow[b]{4}{*}{ Subject } & \multicolumn{9}{|c|}{ Indicators } & \multirow{4}{*}{$\begin{array}{c}\text { Behaviora } \\
\text { State }\end{array}$} \\
\hline & & \multicolumn{5}{|c|}{ Nonverbal Domain } & \multicolumn{4}{|c|}{ Verbal Domain } & \\
\hline & & \multicolumn{3}{|c|}{$\begin{array}{l}\text { Positive } \\
\text { Valence }\end{array}$} & \multirow{2}{*}{$\begin{array}{l}\text { Negative } \\
\text { Valence } \\
\text { (ER) }\end{array}$} & \multirow[b]{2}{*}{ Index* } & \multirow{2}{*}{$\begin{array}{c}\text { Negative } \\
\text { Valence }\end{array}$} & \multicolumn{2}{|c|}{$\begin{array}{l}\text { Positive } \\
\text { Valence }\end{array}$} & \multirow[b]{2}{*}{ Index $\dagger$} & \\
\hline & & $\mathrm{HN}$ & $\mathrm{BC}$ & $\mathrm{EG}$ & & & & Agree & PS & & \\
\hline \multirow[t]{3}{*}{$t_{0}$} & $i$ & 1 & 0 & 1 & 1 & 2,1 & 3 & 0 & 0 & 3,0 & $2,1,3,0$ \\
\hline & $j$ & 1 & 1 & 1 & 1 & 3,1 & 4 & 0 & 0 & 4,0 & $3,1,4,0$ \\
\hline & $k$ & 0 & 1 & 0 & 0 & 1,0 & 1 & 1 & 1 & 1,2 & $1,0,1,2$ \\
\hline \multirow[t]{3}{*}{$t_{1}$} & $i$ & 1 & 1 & 0 & 0 & 2,0 & 1 & 1 & 1 & 1,2 & $2,0,1,2$ \\
\hline & $j$ & 0 & 1 & 0 & 0 & 1,0 & 2 & 0 & 0 & 2,0 & $1,0,2,0$ \\
\hline & $k$ & 0 & 0 & 1 & 1 & 1,1 & 1 & 1 & 0 & 1,1 & $1,1,1,1$ \\
\hline \multirow[t]{3}{*}{$t_{2}$} & $i$ & 0 & 1 & 1 & 1 & 2,1 & 1 & 1 & 0 & 1,1 & $2,1,1,1$ \\
\hline & $j$ & 0 & 0 & 0 & 0 & 0,0 & 5 & 0 & 0 & 5,0 & $0,1,5,0$ \\
\hline & $k$ & 1 & 1 & 0 & 0 & 2,0 & 1 & 1 & 1 & 1,2 & $2,0,1,2$ \\
\hline \multirow[t]{3}{*}{$t_{3}$} & $i$ & 0 & 1 & 1 & 0 & 2,0 & 2 & 0 & 0 & 2,0 & $2,0,2,0$ \\
\hline & $j$ & 0 & 1 & 0 & 1 & 1,1 & 2 & 0 & 0 & 2,0 & $1,1,2,0$ \\
\hline & $k$ & 0 & 1 & 1 & 1 & 2,1 & 1 & 1 & 1 & 1,2 & $2,1,1,2$ \\
\hline
\end{tabular}

Note--HN, head nod; BC, back channel; EG, eye gaze; ER, eye roll; PS, problem solution. Nominal scoring was used, except for verbal negative, for which quantitative scoring (1-5) was used. *Reflects the sum of the summed nonverbal positives and the single nonverbal negative. 'Reflects the quantitative verbal negative and the summed nominal verbal positives. 
sense, since one would construct a test with coefficient alpha across items as the index, but rather, that the investigator assumes that the composite of codes necessarily forms a conceptual singularity. Generally, the number of indicators per domain is so small (e.g., 1-4) and scoring variability so high that traditional reliability analysis (Kline, 1986) is precluded.

\section{Behavioral State}

Whereas a behavioral domain provides an index of $n$ indicators within a subject, the behavioral state is an array of behavioral domain values within a subject (see Table 3). The behavioral state encompasses the behavior of an interactant and produces an interpretable behavioral array. Stated differently, it is a vector, an $n$-tuple of real numbers in an $n$-dimensional euclidean space. In this form, it represents the individual's behavior state in the behavioral process at $t_{x}$. It is the interactant's signature. Although it might appear to have little utility beyond simply being a collection of domain values, it is unique, because the investigator can alter its composition, depending on the research question. Being a dynamic rather than a static representation of the interactant, it potentially can change the calculated intersubject process by adding or removing specific domains (i.e., by adding or removing information to the process). The inclusion or removal of a domain is analogous to inserting or removing codes during data analysis, a procedure common in behavioral observation research when the investigator is trying to find the behavior(s) that most parsimoniously captures the process. The analysts may want to remove a domain that does not contribute uniquely to the overall behavioral state. Intercorrelations among domains may be used in conjunction with theory to determine the best domain set. Domains that do not contribute some independent information create a wasteful conceptualization of the process and should be removed from the behavioral state array. Conceptually, this is similar to general logit modeling, where the objective is to find the best-fitting yet most parsimonious model of the data (see DeMaris, 1992; Kleinbaum, 1994).

\section{Behavioral Process}

When combining behavioral states across subjects, the investigator has two options-retain either a domain-bydomain comparison, or collapse over domains into a single process index. For example, assume that 2 subjects, $i$ and $j$, are each measured on three domains $(A-C)$, with their scoring being $1,3,3$ and $4,3,1$, respectively. This decision is illustrated as

$$
\text { (A: } \left.1_{i} \leftrightarrow 4_{j} \text {; B: } 3_{i} \leftrightarrow 3_{j} \text {; C: } 3_{i} \leftrightarrow 1_{j}\right) t_{x} \text { vs. } y t_{x},
$$

with $y$ representing the process index and $\mathrm{A}-\mathrm{C}$ representing the domains.

By retaining a domain-by-domain comparison, an array of domain values across subjects is available for analysis. Consequently, there is less information loss, and multiple data points are available to illustrate and animate the observed process. Using the domain level data would be most appropriate when the investigator is focusing on specific behavior(s) that have prominence in the research question, a distinction usually guided by theory. In effect, staying at the domain level allows the investigator to ignore other aspects of the interaction. These ignored aspects may not be relevant at that point in time, or the investigator may be systematically looking at the relative contribution of each major domain to the behavioral trajectory. For example, in married couple interaction, negativity is the best predictor of marital quality (Weiss \& Heyman, 1997), yet many, if not most, observational laboratories also code for positive behavior. By using a domain level analysis, the researcher can investigate the relative contribution of each domain; then, he or she can add or remove indicators, or the weighted value assigned to an indicator can be adjusted as data analyses determine its ability to predict behavioral trajectories.

Conversely, the investigator can collapse across domains to create a single index that provides a nice summary indicator of the process. A summary index is more useful for traditional statistical analysis, because it is more tractable than the domain-by-domain comparison. Time series analysis, for example, can trace the behavioral trajectory over time. If the study requires a group comparison, the averaged index score, over some unit of time, is an interpretable behavioral process score for any particular dyad or unit. Group comparisons over multiple, discrete time units can be analyzed in a repeated measures design.

A method of obtaining the summary index is given immediately below; the domain-by-domain method is given in the section of this article on animation.

\section{COORDINATION INDEX}

After each individual is coded (i.e., the indicators), behaviors are summarized (i.e., the domain) and a profile is created (i.e., the state), the object is to conglomerate the disparate across-subjects data strings in such a way that their aggregate reflects the observed process. If we assume that dynamic processes reflect interactant coordination evolving through time and space, such an aggregate must have at least three features: It must show fidelity to the process being observed, be time sensitive, and be interpretable. We interpret interaction along some dimension of organization, typically describing the observed interaction with phrases like "it seems haphazard or disjointed" versus "they seem to act as one." Such descriptions convey our expectations about processes unfolding over time. This expected unfolding reflects an assumption of the underlying coordination among interactants.

At the simplest level, coordination refers to how well "things" work together. For the data summary method proposed herein, this can be further reduced to two perspectives of the same concept: the disparity between 2 or more interactants (this is distance) and the congruous behavior of 2 or more interactants (this is coherence). Dis- 
tance is the disparity between the identified points on 1 object (i.e., the subject) and those on 1 or more other objects. In the method proposed here, these points are represented by domain values, which, as was noted above, represent specific behavioral indicators purported to be essential to the interaction process. Distance, then, is the difference between 2 or more subjects for each of the selected domains.

Coherence is simply the reciprocal of this value. Coherence is a term commonly used in physics to express the coordination between two or more bodies (be they particles or planets) over time and space (see, e.g., Cambel, 1993; Lindley, 1996; Strogatz, 1994).

Coordination, either distance or coherence, assessed between interactants is a proxy of the observed process. Depending on code construction and domain composition, coherence or distance conveys how well or how poorly the process is occurring, relative to expectation.

These two perspectives provide equally valid impressions of interactant coordination. Differences exist primarily in the research question. These concepts of coordination are essentially the same, but interpretation varies by the question asked and by how the indicators, domains, and states are constructed to address the question. For example, if the research question focuses on coordination, such as mother-infant synchronicity during feeding, a natural and interpretable index would be coherence-the interpretation would be reflective of coordination. Conversely, if the research question focuses on differences, such as gender nonverbals during a conversation, distance is the most appropriate (i.e., interpretable) index form.

If one assumes that distance reflects disparity, a simple method of assessing how interactants differ across joint domains is simply to determine their euclidean distance. This is the basic formula used in geometry to determine the absolute distance between two objects, where domain values represent points on the object. Thus, any number of domain states can be used to estimate the disparity between subjects. From this, one gets an absolute value of the disparity between subjects at time $t_{x}$. For example, distance between 2 interactants $(1,2)$ measured on three domains $(\mathrm{A}-\mathrm{C})$ would be estimated as

$$
\begin{aligned}
\text { Distance }_{12} & =|12| t_{x} \\
= & \sqrt{\left.\left(1_{A}-2_{A}\right)^{2}+\left(1_{B}-2\right)_{B}\right)^{2}+\left(1_{C}-2_{C}\right)^{2}} .
\end{aligned}
$$

For 2 interactants, the general formula for distance is

$$
|i j|_{t_{x}}=\sqrt{\sum_{q=1}^{n}\left(i_{q}-j_{q}\right)^{2}},
$$

where $i j$ are interactants and $q$ is domains. ${ }^{3}$

To get coherence, one simply takes the reciprocal of the distance. This provides a measure of congruent action among interactants. It is calculated as

$$
|i j|_{t_{x}}=\frac{1}{\sqrt{\sum_{q=1}^{n}\left(i_{q}-j_{q}\right)^{2}}} .
$$

It can be seen that the denominator is distance and, again, $i j$ are the interactants and $q$ is domains. Note that, if distance equals 0 , a constant must be added to the denominator before calculating coherence.

It should be apparent to the reader that some information loss occurs in creating this multidomain coordination index. However, the inherent flexibility in the way the index is constructed somewhat offsets this loss. For example, it is very easy to construct a coordination index for each domain of interest. An investigator can also model the process differently by rearranging indicator scoring or by adding, dropping, or modifying domains. This allows multiple indices, each reflecting a different feature of the process.

Finally, estimates for distance and coherence are obtainable irrespective of group size. One simply determines the difference between all possible pairings to derive an overall estimate. For example, distance for a 3-interactant process at $t_{x}$ is estimated as

$$
\begin{aligned}
& |i j k|_{t_{x}}= \\
& \quad \sqrt{\left(\delta_{i j t_{x}}-\delta_{i k_{x}}\right)^{2}+\left(\delta_{i j t_{x}}-\delta_{j k_{t_{x}}}\right)^{2}+\left(\delta_{i k_{t_{x}}}-\delta_{j k_{x}}\right)^{2}}
\end{aligned}
$$

where $i j k$ are interactants and $\delta$ is distance derived from Equation 2 above. Again, coherence is $1 /$ distance, where distance is nonzero. If the group being observed is larger than three, the formula becomes more complicated but is still relatively simple (see the Appendix).

Table 4, using data from Table 3, shows the coordination index (distance) derived from subject state values. Note that, in addition to the coordination index, each domain receives an index value at each time unit. As was noted earlier, the investigator elects which domains make up the larger composite index. This allows the investigator to determine the domains used to model the process. ${ }^{4}$

\section{DATA TYPES}

Thus far, we have described a general method for coding behaviors that allow subsequent estimates of coordination among the observed subjects. Interpreting coordination depends on the initial code definitions; this seems self-evident, yet interpretation also depends on the relationship between domains among interactants. Ideally, the investigator has an idea, constructed either empirically or theoretically, of how the interactants are expected to engage each other and how the domains should reflect these assumptions.

Also, it is not necessary to construct domains similarly across subjects. A strength of the proposed method is that the domains could or should be constructed differently for each subject, each construction reflecting the investigator's assumptions about the underlying process and how each interactant affects that process.

Nonuniform scoring of indicators or domains across subjects depends on several assumptions held by the investigator. First, the investigator should determine whether the observed subjects engage in a process that has a clear objective (e.g., nest building, courtship). If so, domains 
Table 4

Coordination Indices Derived From the Behavioral States of 3 Subjects Across Four Domains and Four Time Units (see Table 3)

\begin{tabular}{|c|c|c|c|c|c|c|c|c|c|c|}
\hline \multirow[b]{2}{*}{ Time } & \multirow[b]{2}{*}{ Subject } & \multicolumn{4}{|c|}{$\begin{array}{c}\text { Behavioral State } \\
\text { by Domain }\end{array}$} & \multicolumn{4}{|c|}{$\begin{array}{l}\text { Coordination Index } \\
\text { by Domain }\end{array}$} & \multirow{2}{*}{$\begin{array}{l}\text { Coordination } \\
\text { Index }\end{array}$} \\
\hline & & A & $\mathrm{B}$ & $\mathrm{C}$ & D & A & B & $\mathrm{C}$ & D & \\
\hline \multirow[t]{3}{*}{$t_{0}$} & $i$ & 2 & 1 & 3 & $0)$ & & & & & \\
\hline & $j$ & 3 & 1 & 4 & 0 & 2.45 & 1.41 & 3.74 & 2.83 & 5.48 \\
\hline & $k$ & 1 & 0 & 1 & 2) & & & & & \\
\hline \multirow[t]{3}{*}{$t_{1}$} & $i$ & 2 & 0 & 1 & 2) & & & & & \\
\hline & $j$ & 1 & 0 & 2 & $0\}$ & 1.41 & 1.41 & 1.41 & 2.45 & 3.46 \\
\hline & $k$ & 1 & 1 & 1 & 1) & & & & & \\
\hline \multirow[t]{3}{*}{$t_{2}$} & $i$ & 2 & 1 & 1 & 1) & & & & & \\
\hline & $j$ & 0 & 1 & 5 & 0 & 2.83 & 1.41 & 5.66 & 2.45 & 6.93 \\
\hline & $k$ & 2 & 0 & 1 & 2) & & & & & \\
\hline \multirow[t]{3}{*}{$t_{3}$} & $i$ & 2 & 0 & 2 & $0)$ & & & & & \\
\hline & $j$ & 1 & 1 & 2 & $0\}$ & 1.41 & 1.41 & 1.41 & 2.83 & 3.74 \\
\hline & $k$ & 2 & 1 & 1 & 2) & & & & & \\
\hline
\end{tabular}

are constructed to record how each subject is or is not fulfilling the objective. This allows their combined activity to be interpretable. For example, if a 2 -interactant process requires that each individual engage in a specific behavior and only one does so, their respective codes would be scored 1 and 0 -implying that the putative process did not occur.

Conversely, if the investigator decides that the process has no clear goal (at least within the period of observation), he or she must anticipate how multiple codes from different interactants fit together to form an interpretable index. Determining how to construct such domains is helped by sorting the codes into two basic types, classified according to how a code for one interactant relates to a comparable code for another interactant.

\section{Symmetrical Indicators}

At the simplest level, indicators (i.e., codes) divide into two classes: symmetrical and complementary. Symmetrical coding occurs when each subject in the interaction has similar obligations to the process. Symmetrical codes are the same for any 2 subjects; consequently, the interpretation is that the subjects are doing the same thing if their scores are the same.

\section{Complementary Indicators}

Complementary codes are the other class of codes. Each code complements the behavior or action of another subject. Metaphorically, these joint behaviors across subjects are similar to a lock and key--together, their respective parts form a whole. For example, suppose that the investigator is coding dyadic conversations among married adults and that a talk turn is the basic unit of time. In every talk turn, one individual is designated as the speaker, and the other is the listener. A number of codes are assigned to speaker and listener roles; many of these codes are very different, yet complement each other. The speaker, for example, should be coded for speaking behaviors, and the listener should be coded for not talking and attending (e.g., facing or eye gazing, back channel).
Together, their behaviors form a whole complementary unit. Although endorsed for different behaviors (talk vs. no talk, attending), the action within that role forms the complement. In effect, a complementary code is $50 \%$ of a unit; its complement must be present for the composite to form.

Finally, it is also possible or, sometimes, necessary to mix symmetrical and complementary codes to construct an interpretable coordination index. This construction is especially appropriate if some interactants engage in parallel behaviors, whereas others in the same field engage in different yet critical behavior. For example, suppose that an interacting dyad shows high coherence that consistently cycles toward violence, much like two males vying for access to a female during courtship. The males would be coded for symmetrical behavior, whereas the recipient female(s) would be coded for complementary behavior. This allows for a coherence index for each pairing of interactants and for the group.

\section{Illustration}

In the remaining section, I illustrate how the data reduction method described above can be used to animate and visualize processes captured by the data. First, I show how the coordination index provides a simple data form familiar to most investigators. Second, and more important, I show how this data reduction method extracts data from a social process and uses these extracted data to explore and possibly analyze the observed process by using scientific visualization techniques.

\section{Coordination Index}

Graphical representation. The coordination index is the traditional data form found in the social and behavioral sciences. Consequently, it lends itself to standard graphical, time series, and sequential data analytic strategies. Specific time series and sequential data analysis techniques are beyond the scope of this paper and can be found elsewhere (see Bakeman \& Quera, 1995; Gottman \& Roy, 1990; Haccou \& Meelis, 1994). 
As a multivariate summary of interaction, the coordination index is especially useful for viewing change over time. Aside from its obvious statistical analytic value, and because we assume that it represents the process, it is visually informative. Changes in slope and level reflect changes in the process. These changes are interpretable on the basis of domain selection and configuration.

The line graph in Figure 2 shows the coordination index, calculated as distance, for an adult couple during a 15 -min conversation. Figure 2 displays only $60 \mathrm{sec}$ (minutes 3-4) of the couple interaction. Three domains were used to construct the index: real-time self-reported affect (positive [1], neutral [0], negative [-1]), magnitude of the reported affect $(0,1,2)$, and duration in the state. Additional information about the acquisition of these data can be found in Griffin (1993).

The graph shows the disparity of the couple's affective response to the conversation. Note that the disparity is lowest from seconds 18 to 34 and again from seconds 40 to 52 . These intervals of reduced disparity show where the couple increased affect coherence. These moments may be rich in information (e.g., unique verbal content) for the investigator studying couple synchronicity or some aspect of coordination. Although this example used a real-time self-report domain (affect), this figure could be similarly constructed using domains such as nonverbal negative, verbal negative, verbal positive, and nonverbal positive.

\section{Scientific Visualization}

Numerous other investigators have proposed methods for quantifying state processes; most are computationally and mathematically quite sophisticated (see, e.g., Thomas \& Malone, 1979) but are seldom used, despite their appropriateness. I have tried to simplify process detection and analysis by minimizing the mathematics involved and depending more on reducing and coordinating available data from standard coding techniques. Moreover, I wanted the output data to be easily integrated into current data visualization and animation techniques. This is important for two reasons. First, the amount and the quality of available observational data are increasing rapidly with advances in audiovisual data collection technology (see, e.g., Noldus, 1991). Second, as the ability to store data increases, investigators gathering these large amounts of data expect a greater return from the data. Central to this expectation is the ability to assess and model complex processes.

Other scientific fields have also had to develop methods for capturing, quantifying, and understanding complex dynamic systems (e.g., hydrology, meteorology). One tool that has rapidly developed to address this need is scientific visualization (also referred to a SciVis). Increasingly powerful computers make it possible to investigate incredibly complex dynamics by using realistic simulations and animations. Data interpretation with visualization and animation techniques has become a major re-

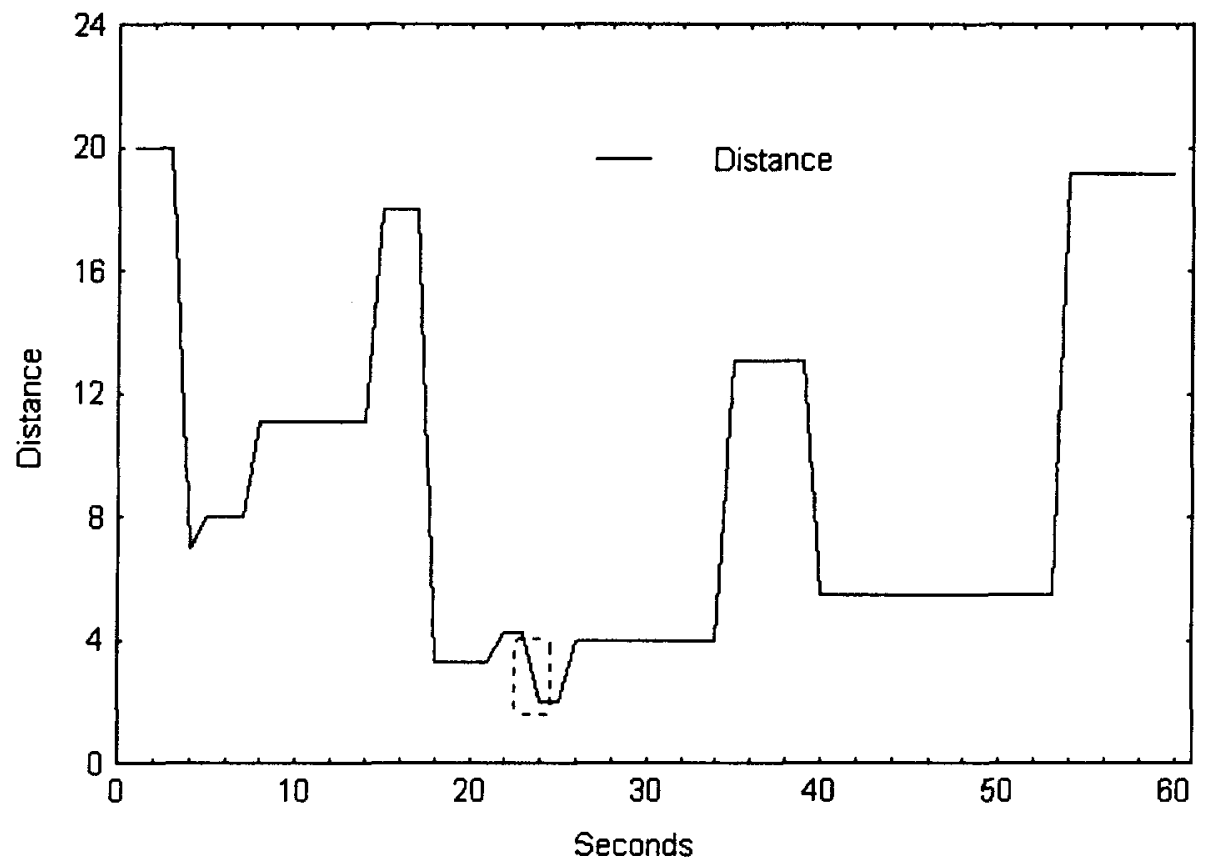

Figure 2. Coordination index (calculated as distance) of $60 \mathrm{sec}$ of data for an adult couple compiled from affect, magnitude of affect, and duration in affect state. The rectangular box at $\simeq 22-23 \mathrm{sec}$ spans Frames 4-6 in Figure 5 (see the text). 
search focus in computer science programs at universities throughout the world. SciVis techniques, either dynamic or static, allow the investigator to observe simultaneous influences on a process in hopes of acquiring a greater understanding of the system that generated the data. SciVis compresses a lot of data into one picture, revealing correlations between different quantities both in space and in time. An introduction to SciVis is beyond the scope of the author and this paper (see Nielson, Hagen, \& Muller, 1997, for a good introduction); instead, I will illustrate how the data reduction method described herein lends itself to visualization and animation techniques that are useful for exploring social processes. These animation techniques consolidate and visualize information, so that patterns or tendencies are seen before analysis, at the exploratory stage. These observations, in turn, lead to better data analytic strategies.

Two-dimensional animation. Before describing the more complex three-dimensional (3-D) animation techniques, I will show how a simpler two-dimensional (2-D) animation provides the researcher with a visual display of the data. Three domains were used in Figure 2: affect state, state magnitude, and state duration. Assume that state magnitude is less important than either affect state or state duration and can be dropped with minimal information loss. To make a graphical representation of the process, we first let a small circle represent each affect state (positive, neutral, and negative). Assume that each affect state circle is color coded (red = negative, neutral = white, and blue $=$ positive). These small circles surround a larger, central circle that represents the couple's joint state. This representation is given in Figure 3.

Using a small indicator to trace transitions in real time, this simple configuration can model the couple's interaction processes for state transition, affect, and affect duration. Specifically, the model animates the movement from state to state for each interactant. If we assume a simple additive model in which the affect state of each interactant contributes equally to the joint process, their combined state at $t_{x}$ is shown by the color of the central circle. I constructed such a model with a small publicly available software package called Samba (Stasko, 1998). ${ }^{5}$

Figure 4 shows the screen after $186 \mathrm{sec}$ of animating the state transitions. Note the labeled circles; each is shaded according to the affect color description above. A transition matrix and a cumulative duration box were included to help the researcher follow the data flow after animation begins. To show transitions, a small black circle flows from the occupied state to the next state at each transition. Although it is difficult to see in shades of gray, at this point Subject $i$ was in neutral (white), and Subject $j$ was in negative (red); hence, the joint state circle was pink. During the period of selected animation, several interesting features of the interaction were evident: Subject $i$ tended to follow Subject $j$ into negative, and Subject $i$ showed much greater movement (i.e., shorter durations, more transitions). These impressions occurred from a simple viewing of the animation. Interpretation could be enhanced by configuring a video setup that would overlay the animation or place it beside the video playback.

Clearly, the purpose of this illustration was not to describe the process between these 2 individuals but, rather, to show that a simple 2-D animation program can model complex processes. By altering domains and the visual presentation of shapes, the researcher can track movement and discern patterns that are elusive when looking only at the raw data.

Three-dimensional animation. Moving beyond 2-D into 3-D animation is a substantial jump, both conceptually and technically. On the technical side, the software and hardware requirements increase dramatically, although advances in computer technology in these areas are making it easier for social and behavioral scientists to access the necessary modeling tools.

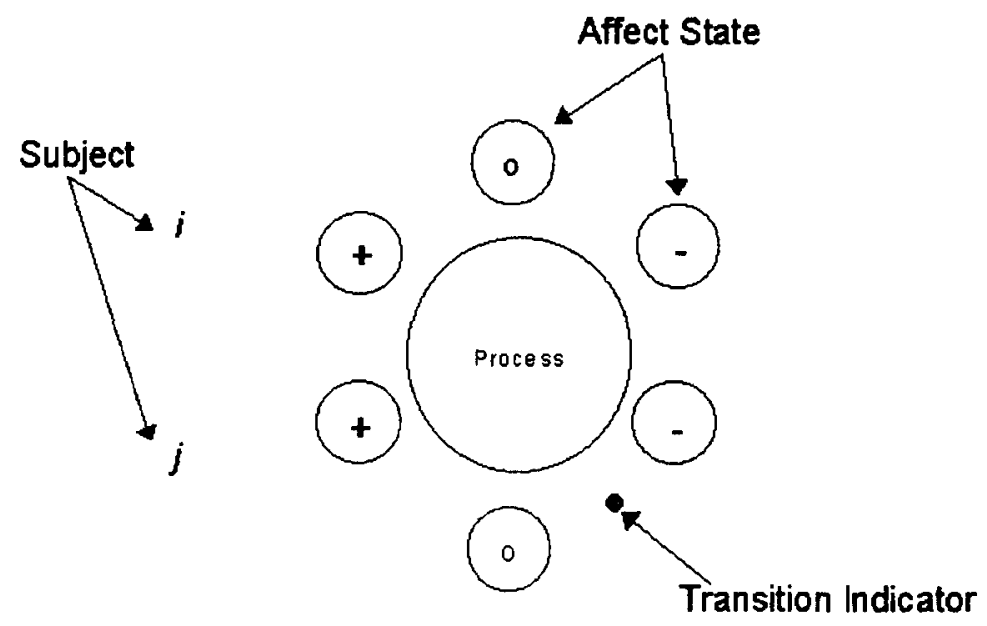

Figure 3. Diagram representing a two-dimensional animation configuration of 2 subjects over three states, showing transitions with a central joint state indicator. 


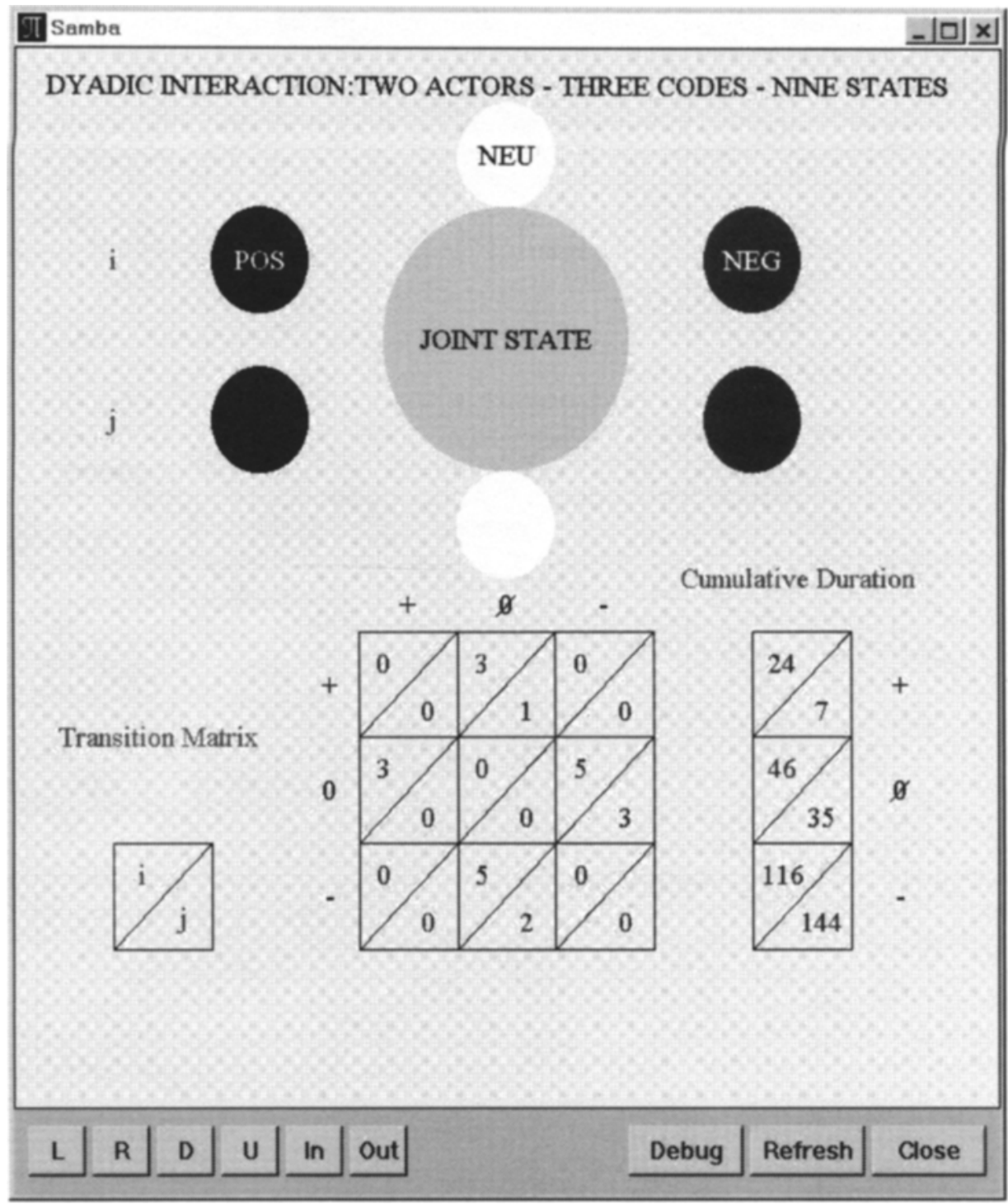

Figure 4. Samba implementation of Figure 3. This two-dimensional animation models the dyadic interaction of a divorced couple. Program view after $186 \mathrm{sec}$ of interaction. Note the cumulative duration and transition matrices.

The conceptual side is more challenging. Consider that each coded feature of a subject represents a plane and that these $n$ planes can be used to describe the "location" (i.e., disposition) of the subject in 3-D space at $t_{x}$. In effect, the planes converge to form a point in space. Consequently, to visually track processes involving numerous subjects, the number of dimensions (i.e., codes) visualized per subject should be low to moderate. Objects representing more than 4-6 domains are difficult to interpret.

Although this section will demonstrate 3-D animation, the number of dimensions being animated can be more than three. For example, if we allow a sphere to represent a subject, only three domains are needed to place the subject in space. These three domains represent $x, y$, and $z-$ in effect, a point in space. However, the researcher could also add color, size, texture, or any other of several fea- tures to the point. Each feature reflects a domain value. It is easy to see that having an $n$-dimensional object in a 3-D animation is very easy to construct but potentially difficult to interpret.

Recall that Figure 2 uses three domains: state magnitude, affect state, and state duration. Allow these three domains to represent dimensions $x, y$, and $z$, respectively. Figure 5 shows six frames extracted from an animation of the same interaction described in the 2-D illustration. Frames 1-3 are $1 \mathrm{sec}$ apart (30 frames per second), and Frames $4-6$ are approximately 15 frames $(0.5 \mathrm{sec})$ apart. The latter frames are closer in time, to show the rapid affect change that occurred at this point in the interaction.

The interactants, $i$ and $j$, are shown as spheres located in space by their respective $x, y$, and $z$ coordinates. In Figure 5 , the coordinate $y$ (i.e., the state) runs horizontally, 

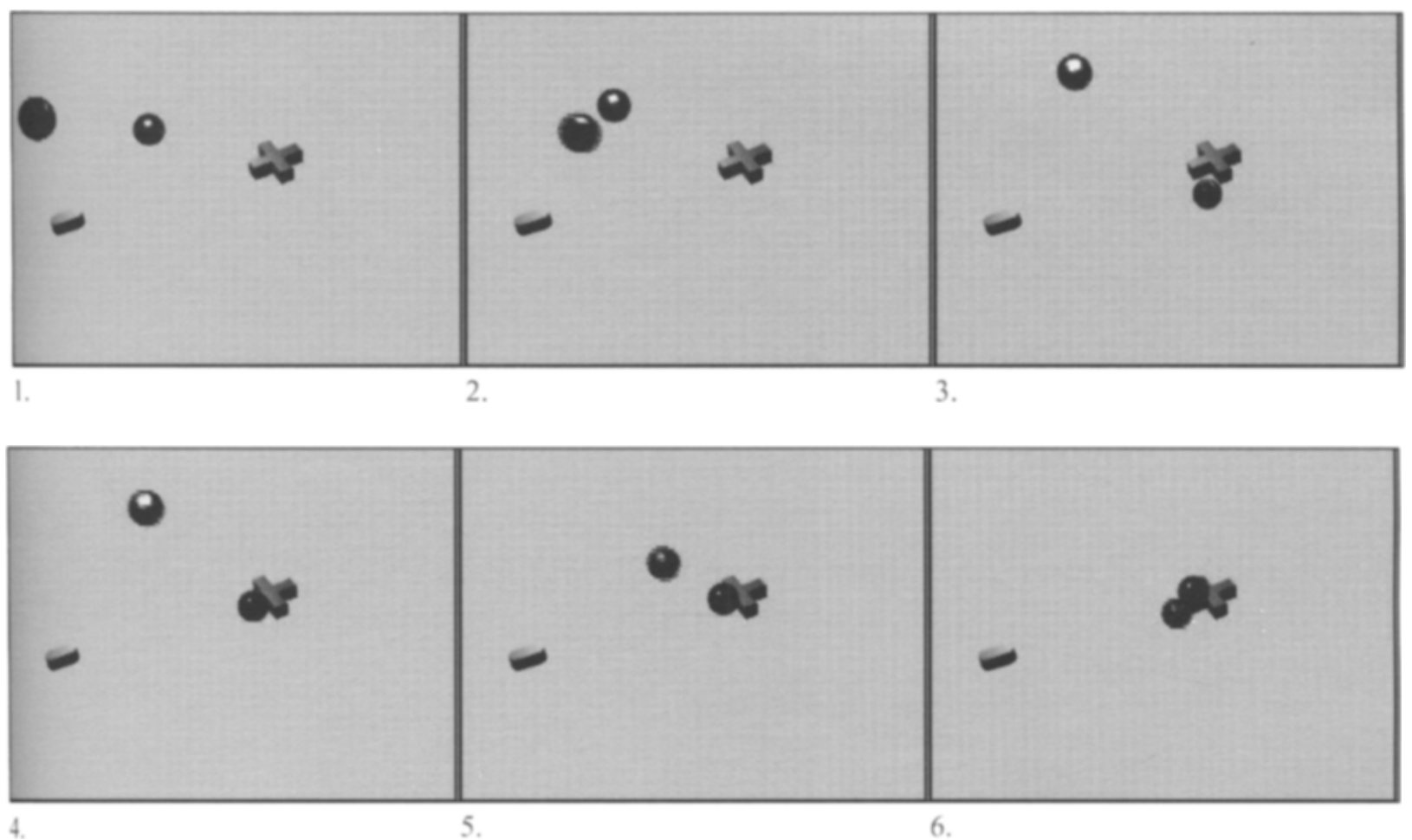

Figure 5. Six seconds (30 frames per second) of a three-dimensional animation of 2 subjects, using affect, affect magnitude, and affect duration as coordinate points. The coordinate $y$ (i.e., state) runs horizontally, $x$ (i.e., magnitude) runs vertically, and $z$ (i.e., state duration) extends toward the viewer. Frames illustrate one partner following the affect lead of the other partner (see the text for additional explanation).

and $x$ (i.e., the magnitude) runs vertically. The $z$ coordinate is state duration and extends toward the reader-that is, the longer the individual stays in state, the more the sphere rises. Positive and negative signs have been placed in space to reference the domain state; neutral is between the two reference signs. Modeling the process occurs by allowing the spheres to move through space in real time. This simple real-time animation sequence illustrates the use of domains to assess process. It shows the subtle features of affect changes between an adult couple having a conversation. During actual animation, the spheres move past each other, up and down, right and left. Sometimes they follow each other, and sometimes they quickly move in opposite directions, all occurring in real time. Animating the data this way permits the analyst to visually search for the patterns. The selected frames in Figure 5 show one partner's affect quickly following the other partner's reported affect. Interestingly, these near-synchronous shifts occurred without either partner's knowing the reported affect of the other person.

In addition, the investigator can increase the animation complexity of this simple model. For example, more domains (e.g., verbal negative, nonverbal negative, nonverbal positive) would add complexity. These additions might include color, size, or shape changes, each new domain representing an assessed dimension of the subject. The researcher could also install visual signals indicating points in the process that are theoretically or empirically important. For example, to indicate severe anger, the sphere could start blinking after the individual resides in high-magnitude negative past a predetermined point.

Finally, because the coordination index is composed of the domains used in the animation, it is possible to track animated movements corresponding to the index value. For example, recall that Figure 2 shows the coordination index (calculated as distance) of the couple over the course of $60 \mathrm{sec}$ of interaction. A part of this interaction is shown in the six frames of Figure 5. The rectangular box spanning seconds $22-23.5$ corresponds approximately to Frames 4-6 of Figure 5. The low distance value is evident in the closeness of the two spheres. Comparing sphere movement with the graphed index changes is easy. In fact, with a sufficiently powerful computer, the graph can be drawn in real time concurrent with the movement of the animation.

This illustration used 2 subjects. All comments and suggestions made in this paper are equally applicable to the interactions of $\geq 3$ interactants.

\section{Learning Visualization and Animation}

Initial forays into data animation can be intimidating for the traditionally trained social and behavioral scientist. Increasingly powerful computers and turnkey graphics and animation programs are markedly decreasing the 
learning curve in this area. I used Samba for the 2-D animation in Figure 3. I selected Samba because it is publicly available, easy to learn and use, and may be sufficient for many investigators, depending on the complexity of their data. As was noted, working in three dimensions is more difficult and requires more computing power. Fortunately, commercially available 3-D modeling and animation software programs are now accessible (i.e., the learning curve is less steep) to most researchers. The animations in Figure 5 were drawn and rendered with TrueSpace4 (TrueSpace4, 1998), a commercial 3-D animation program. The completed animation is in AVI format and is viewable on any standard PC. Commercial 3-D programs are available for PC, Mac, and UNIX systems, ranging in price from several hundred to several thousand dollars, and are available at most computer stores. UNIX- or Xwindowsbased programs (e.g., PV-Wave from Visual Numerics, 1998; also available for Windows) tend to be more costly and powerful but are also more likely to be available though university departments (i.e., computer science) or visualization centers. Many universities have departments, programs, or centers that specialize in scientific visualization; these would be good places to learn visualization techniques, especially with UNIX-based programs. Information about the PC-based systems is available in trade magazines (e.g., PC Magazine; $D V$ ), where the various programs are reviewed and rated by cost and function.

\section{CONCLUSION}

This article introduced a method of taking observational data from multiple subjects, reducing it, and combining it into an array of values or a single index. This index attempts to preserve the integrity of the data while providing a mechanism for modeling social processes. With advances in audiovisual technology, more and better observational data are available for analysis and process modeling. The method proposed herein is also a call to model the observed social processes by using recently developed visualization techniques.

\section{REFERENCES}

AldRICH, J. H., \& Nelson, F. D. (1984). Linear probability, logit, and probit models (Sage University Paper series on Quantitative Applications in the Social Sciences, 07-045). Beverly Hills, CA: Sage.

Als, H., Tronick, E., \& Brazelton, T. (1979). Analysis of face-toface interaction in infant-adult dyads. In M. Lamb, S. Soumi, \& G. Stephenson (Eds.), Social interaction analysis: Methodological issues (pp. 33-76). Madison: University of Wisconsin Press.

Bakeman, R., \& GotTMAN, J. M. (1997). Observing interaction: An introduction to sequential analysis (2nd ed.). New York: Cambridge University Press.

Bakeman, R., \& QUeRa, V. (1995). Analyzing interaction: Sequential analysis with SDIS and GSEQ. New York: Cambridge University Press.

Bernieri, F. J., Reznick, J. S., \& Rosenthal, R. (1988). Synchrony, pseudo-synchrony, and dissynchrony: Measuring the entrainment process in mother-infant interactions. Journal of Personality \& Social Psychology, 54, 243-253.

Brogan, D. C., \& Hodgins, J. K. (1997). Group behaviors for systems with significant dynamics. Autonomous Robots, 4, 137-153.

CAIRNS, R. B. (1979). The analysis of social interactions: Methods, issues, and illustrations. Hillsdale, $\mathrm{NJ}$ : Erlbaum.
Cambel, A. B. (1993). Applied chaos theory: A paradigm for complexity. San Diego: Academic Press.

COHEN, J. (1960). A coefficient of agreement for nominal scales. Educational \& Psychological Measurement, 20, 37-46.

DeMarIS, A. (1992). Logit modeling (Sage University Paper series on Quantitative Applications in the Social Sciences, 07-086). Beverly Hills, CA: Sage.

FOGEL, A. (1993). Developing through relationships: Origins of communication, self, and culture. Chicago: University of Chicago Press.

Fogel, A., LyRA, M. C., \& VAlsiner, J. (1997). Dynamics and indeterminism in developmental and social processes. Mahwah, NJ: Erlbaum.

Gardner, W. (1995). On the reliability of sequential data: Measurement, meaning, and correction. In J. M. Gottman (Ed.), The analysis of change (pp. 339-359). Mahwah, NJ: Erlbaum.

GotTMAN, J. M. (1994). What predicts divorce? The relationship between marital processes and martial outcomes. Hillsdale, $\mathrm{NJ}$ : Erlbaum.

GotTman, J. M., \& Roy, A. K. (1990). Sequential analysis: A guide for behavioral scientists. New York: Cambridge University Press.

Griffin, W. A. (1993). Transitions from negative affect during marital interaction: Husband and wife differences. Journal of Family Psychology, 6, 230-244.

Griffin, W. A., \& GotTMAn, J. M. (1990). Statistical methods for analyzing family interaction. In G. R. Patterson (Ed.), Family social interaction: Content and methodology issues in the study of aggression and depression (pp. 130-168). Hillsdale, NJ: Erlbaum.

Haccou, P., \& MeELIS, E. (1994). Statistical analysis of behavioural data: An approach based on time-structured models. Oxford: Oxford University Press.

Hinde, R. A. (1997). Relationships: A dialectical perspective. Hove, U.K.: Psychology Press.

Hinde, R. A., \& Herrmann, J. (1975). Frequencies, durations, derived measures and their correlations in studying dyadic and triadic relationships. In H. R. Schaffer (Ed.), Studies in mother-infant interaction (pp. 19-46). London: Academic Press.

Hinde, R. A., \& Stevenson-Hinde, J. (1987). Interpersonal relationships and child development. Developmental Review, 7, 1-21.

Hosmer, D. W., \& Lemeshow, S. (1989). Applied logistic regression. New York: Wiley.

Kleinbaum, D. G. (1994). Logistic regression. New York: SpringerVerlag.

KLINE, P. (1986). A handbook of test construction. New York: Methuen.

Lamb, M., Suomi, S., \& Stephenson, G. (1979). Social interaction analysis: Methodological issues. Madison: University of Wisconsin.

LiNDLEY, D. (1996). Where does the weirdness go? New York: Basic Books.

Markman, H. J., \& NotaRius, C. I. (1987). Coding marital and family interaction: Current status. In T. Jacob (Ed.), Family interaction and psychopathology: Theories, methods, and findings (pp. 329-390). New York: Plenum.

Martin, P., \& Bateson, P. (1996). Measuring behaviour: An introductory guide (2nd ed.). Cambridge: Cambridge University Press.

Newell, K. M., \& MolenaAR, P. C. (1998). Applications of nonlinear dynamics to developmental process modeling. Mahwah, $\mathrm{NJ}$ : Erlbaum.

Nielson, G. M., Hagen, H., \& Muller, H. (1997). Scientific visualization: Overviews, methodologies, and techniques. Los Alamitos, CA: IEEE Computer Society Press.

Noldus, L. P. J. J. (1991). The Observer: A software system for collection and analysis of observation data. Behavior Research Methods, Instruments, \& Computers, 23, 415-429.

Noller, P. (1984). Nonverbal communication and marital interaction. New York: Pergamon.

Northey, S., Griffin, W. A., \& Krainz, S. (1998). A partial test of the psychosomatic family model: Marital interaction patterns in asthma and nonasthma families. Journal of Family Psychology, 12, 220-233.

PV-WAVE [Computer software] (1998). Houston, TX: Visual Numerics.

REYNOLDS, C. (1987). Flocks, herds, and schools: A distributed behavioral model. Computer Graphics, 21, 25-34.

Rogosa, D., \& GHANDour, G. (1991). Statistical models for behavioral observations. Journal of Educational Statistics, 16, 157-252.

SACKETT, G. P. (1978). Measurement in observational research. In G. P. 
Sackett (Ed.), Observing behavior: Vol. 2. Data collection and analysis methods (pp. 25-43). Baltimore: University Park Press.

SHaw, E. (1970). Schooling in fishes: Critique and review. In L. Aronson, E. Tobach, D. Leherman, \& J. Rosenblatt (Eds.), Development and evolution of behavior: Essays in memory of T. C. Schneirla (pp. 452-480). San Francisco: Freeman.

StaSko, J. T. (1998). Samba [Computer software]. Atlanta: Georgia Institute of Technology, College of Computing.

Strogatz, S. H. (1994). Nonlinear dynamics and chaos. Reading, MA: Perseus Books.

SUEN, H., \& ARY, D. (1989). Analyzing quantitative behavioral observation data. Hillsdale, NJ: Eribaum.

Thomas, E. A. C., \& Malone, T. W. (1979). On the dynamics of twoperson interactions. Psychological Review, 86, 331-360.

TrueSpaCe4 [Computer software] (1998). Mountain View, CA: Caligari Corporation.

VAN GeErT, P. (1994). Dynamic systems of development: Change between complexity and chaos. New York: Harvester Wheatsheaf.

Weiss, R. L., \& Heyman, R. E. (1997). A clinical-research overview of couples interactions. In W. K. Halford \& H. J. Markman (Eds.), Clinical handbook of marriage and couples intervention (pp. 13-41). New York: Wiley.

WICKENS, T. (1989). Multiway contingency tables analysis for the social sciences. Hillsdale, $\mathrm{NJ}$ : Erlbaum.

\section{NOTES}

1. An indicator may not occur when observed over a short interval of time, making it impossible to calculate a ratio. To create a ratio without an occurrence, a constant is usually added (e.g., .05) to the numerator and denominator. This somewhat distorts reality, because a value has been assigned to a nonoccurrence. In general, the smaller the constant (e.g., .5 vs. .05 ), the better the fit to the expected ratio if there was an occurrence of the behavior; however, with no occurrence, the very small constant constructs very large odds. This is acceptable if the investigator is aware of the effects of the constant and applies it equally across subjects. This is not a problem with quantitative indicators, because they take on values of $\geq 1$.

2 . By taking the natural log of the odds, the estimated value becomes symmetrical around zero - that is, the value is comparable in magnitude, irrespective of valence. This provides a better interpretation of the relationship between the two dimensions of the domain, because the quantity varies from $-\infty$ to $\infty$, with no difference equaling 0 (see Wickens, 1989). An example of using logit modeling with observational data can be found in Northey, Griffin, and Krainz (1998). A good general reference is Hosmer and Lemeshow (1989) or Aldrich and Nelson (1984).

3. There are a number of ways in which to construct the index, once the differences have been calculated, and several of these methods were examined during the development of this paper. One possible alternative to the overall difference score is the averaged pairwise difference. However, the averaged pairwise difference loses some information, as compared with the overall difference. For example, in Table 4, Domains $A$ and $D$ have values $2,3,1$ and $0,0,2$, respectively. The averaged pairwise difference for both is 1.33 , despite their differing values. Conversely, the overall distance index (as shown in Table 4) produced a value for Domain A of 2.45 and of 2.83 for Domain $D$; coherence values are .41 and .35 , respectively. The lower distance and higher coherence of Domain A (relative to Domain D) are consistent with the distribution of values within the domain and more accurately reflect the intent of the index.

4. Values for Table 4 were calculated by using a simple spreadsheet formula, Microsoft Excel (Office 97). For 3 subjects over four domains, the formula was $=$ SQRT $\left[(\mathrm{B} 2-\mathrm{B} 3)^{2}+(\mathrm{B} 2-\mathrm{B} 4)^{2}+(\mathrm{B} 3-\mathrm{B} 4)^{2}+\right.$ $(\mathrm{C} 2-\mathrm{C} 3)^{2}+(\mathrm{C} 2-\mathrm{C} 4)^{2}+(\mathrm{C} 3-\mathrm{C} 4)^{2}+(\mathrm{D} 2-\mathrm{D} 3)^{2}+(\mathrm{D} 2-\mathrm{D} 4)^{2}+$ $\left.(\mathrm{D} 3-\mathrm{D} 4)^{2}+(\mathrm{E} 2-\mathrm{E} 3)^{2}+(\mathrm{E} 2-\mathrm{E} 4)^{2}+(\mathrm{E} 3-\mathrm{E} 4)^{2}\right]$, where [Subject 1 Domain 1] resides in cell B2, [Subject 2 Domain 1] in B3, and so forth. This pattern continues until the last relevant cell--[Subject 3 Domain 4]--in Cell E4.

5. Developed at Georgia Tech, Samba is an interactive animation interpreter and generator that has batch mode capacity. It uses ACSII commands, one command per line. The simplicity of the software permits the researcher to construct basic animated models without programming.

\section{APPENDIX}

The formula for distance easily expands to include $\geq 3$ subjects. Assume $m$ domain values per subject, each reflecting $\geq 1$ indicator(s). For 3 subjects (1-3), this is illustrated by

$$
\begin{aligned}
& 1: 2,3,1,4,2,2, \ldots m \\
& 2: 1,1,4,2,1,4, \ldots m \\
& 3: 4,1,1,2,4,3, \ldots m .
\end{aligned}
$$

Allowing $\delta$ to represent the calculated distance between any 2 interactants (see Equation 2), a 3-person coordination index is derived by

$$
|123|_{t_{x}}=\delta_{123_{t_{s}}}=\sqrt{\delta_{12_{A}}^{2}+\delta_{13_{A}}^{2}+\delta_{23_{A}}^{2}+\delta_{12_{B}}^{2}+\delta_{13_{B}}^{2}+\ldots+\delta_{23_{M}}^{2}} .
$$

The subscript for time has been removed on the right side of the equation to improve clarity; each point of calculation is at time $t_{x}$, as is shown on the left side of the equation.

Moving beyond 3 interactants, this formula is generalized to

$$
|123 \ldots n|_{t_{x}}=\sqrt{\sum_{\substack{i<j \\ i=1 . \ldots, n \\ j=2 \ldots, n}} \delta_{i j}^{2}},
$$

where $123 \ldots n$ on the left side of the equation represents the number of subjects and $\delta_{i j}$ represents the distance calculation between any 2 subjects for a specific domain. Note the subscripting showing $i$ preceding $j$; this ensures that duplicate comparisons are not included in the calculations. The order of comparisons shown in Equation Al above clearly illustrates this.

(Manuscript received August 23, 1999; revision accepted for publication October 3, 1999.) 